\title{
System Evacuation Metrics Collector for IGP and cryo-cycle performance management (SEMCi)
}

Lambertus Alink ${ }^{1}$, Clint Potter $^{2}$, Bridget Carragher ${ }^{2}$, Edward Eng ${ }^{2}$, Kashyap Maruthi ${ }^{3}$, Anchi Cheng ${ }^{3}$ and Robert Gheorghita ${ }^{1}$

${ }^{1}$ NYSBC, United States, ${ }^{2}$ New York Structural Biology Center, New York, New York, United States, ${ }^{3}$ New York Structural Biology Center, United States

Cryo-electron microscopy (cryo-EM) is transforming rapidly to be the preferred tool for routine determination of protein structures at near atomic resolution. This development has substantially increased the number of biomedical researchers requesting access to high-end instrumentation. The high costs and long wait times for access push facilities to maximize instrument availability and prevent unnecessary heat-up cycles and unplanned maintenance where time consuming vacuum issues need to be solved. To meet high availability goals, we have developed a System Evacuation Metrics Collector for automated IGP and cryo-cycle use (SEMCi).

On getting the electron microscope column and octagon (the area where the cold sample is positioned) to ultra-high vacuum one of the main obstacles to overcome is water vapor. The pumping speed for water is initially high because formed layers in the microscope will have weaker water-to-water bonds than the layers closer to the chamber wall. With system ageing the desorption rate will drop as the stronger-bonded molecules are exposed ${ }^{[1]}$. From the fundamental relationship for desorption rate $(\mathrm{Q})$ equaling pumping speed $(\mathrm{S}) \times$ pressure $(\mathrm{P})$, it is easy to infer that the desorption rate needs to fall to some given point before a given pressure can be achieved with a fixed pumping speed. Typical cryo-EM microscopes have surfaces in the vacuum system that are cooled to $\mathrm{LN}_{2}$ temperature during operation. The water in the column will absorb on these surfaces trapping multiple layers over time. During a so called cryo-cycle the cold surface is heated up to expel the water from the column. The setup presented here consists of two steps. In the first step the Pfeiffer Prisma Plus residual gas analyzer (RGA) ${ }^{[2]}$ is set to continuously analyze the amount of leftover gasses in the vacuum chamber. Its output is saved and in the second step sent to a so-called Raspberry PI single board computer ${ }^{[3]}$. The Pi uses an intelligent and autonomous python script to process the data automatically and in real time. Both steps can be automated and controlled by the PI depending on the model RGA.

Most interesting is to check the water content (mass 18) in the vacuum during a heat up cycle. Once the water content is below a certain threshold the heat up cycle can be stopped and the IGP's can be started by triggering the microscope through its standard scripting interface. An email can be sent to the operator that data collection can be started. In addition, the molecular mass 28 and $32\left(\mathrm{~N}_{2}\right.$ and $\left.\mathrm{O}_{2}\right)$ can be monitored and detection can serve as early warning signs for vacuum problems.

We will present the design of the system and show how much the implementation can help improve system availability and efficiency at the Simons Electron Microscopy Center, home to 12 TEMs. 

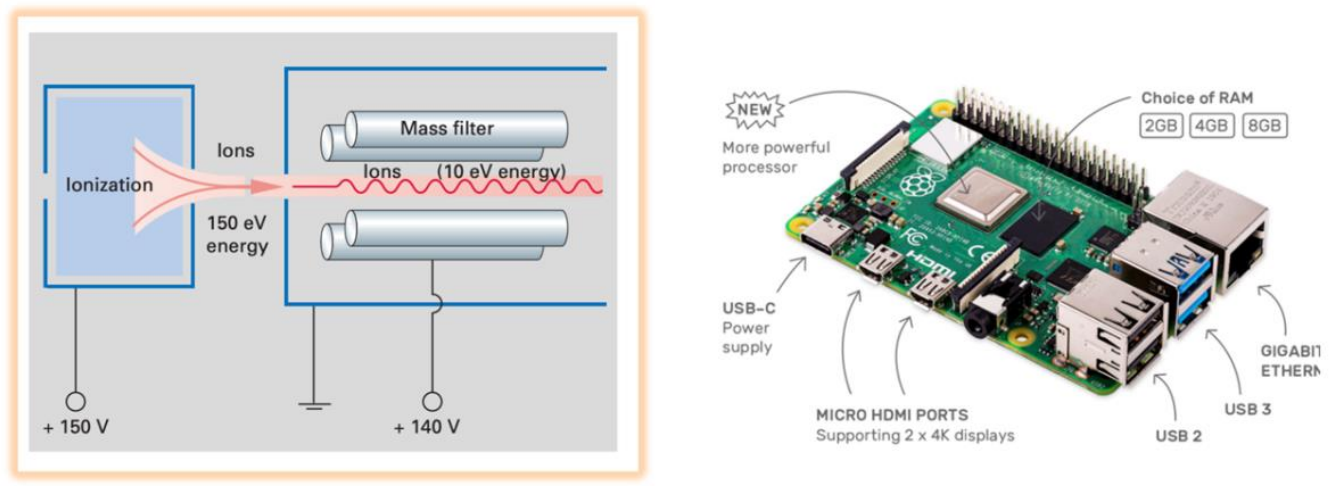

Figure 1. Left: Schematic working of the Pfeiffer residual gas analyzer (RGA). Residual gases are ionized, and the mass filter counts the molecules for a certain mass in a certain time. Right: Raspberry PI single board PC that is used to automatically process the RGA data and control the microscope by scripting using Python. Optionally values can be stored to a server monitor network.

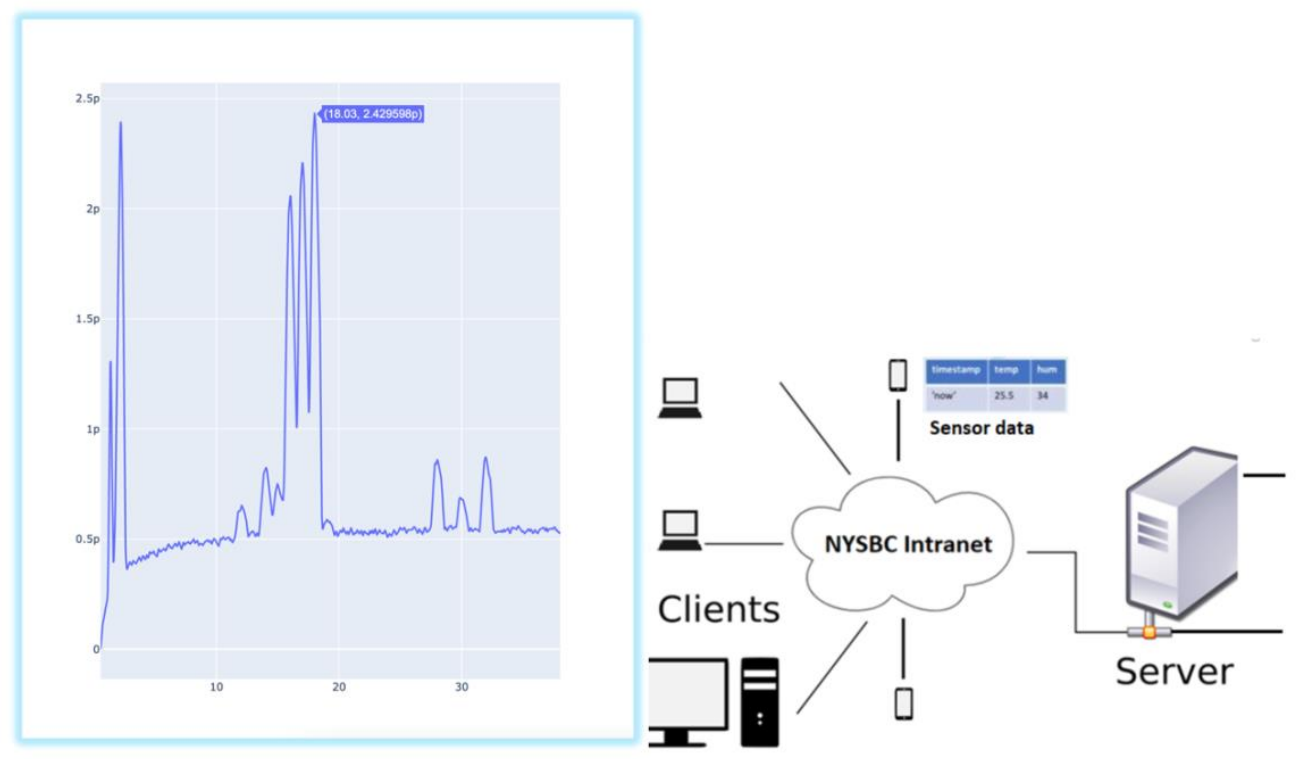

Figure 2. Left: Webpage showing residual gas analysis data. Horizontal is mass. Vertical is ion count. Note that mass 18 is $\mathrm{H}_{2} \mathrm{O}$. Raspberry PI performs autonomous data analysis and real time vacuum monitoring. Alerts can be set up. Right: Architecture of the existing sensor monitor network. Clients are connected to a central server for data storage. of the internal working of a Pfeiffer residual gas analyzer.

\section{References}

${ }^{[1]}$ https://www.mtm-inc.com/av-20101012-water-vapor-in-vacuum-systems.html

${ }^{[2]}$ https://www.pfeiffer-vacuum.com/productPdfs/PTM06241111.en.pdf

${ }^{[3]}$ https://www.raspberrypi.org/ 